Article

\title{
Variation in Dehydration Tolerance, ABA Sensitivity and Related Gene Expression Patterns in D-Genome Progenitor and Synthetic Hexaploid Wheat Lines
}

\author{
Yumeto Kurahashi *, Akihiro Terashima * and Shigeo Takumi * \\ Laboratory of Plant Genetics, Graduate School of Agricultural Science, Kobe University, Nada-ku, \\ Kobe 657-8501, Japan; E-Mails: 096a512a@stu.kobe-u.ac.jp (Y.K.); at3818@yahoo.co.jp (A.T.) \\ \# These authors contributed equally to this work \\ * Author to whom correspondence should be addressed; E-Mail: takumi@kobe-u.ac.jp (S.T.); \\ Tel. +81-78-803-5860; Fax: +81-78-803-5859
}

Received: 22 May 2009; in revised form: 16 June 2009 / Accepted: 16 June 2009 /

Published: 18 June 2009

\begin{abstract}
The wild wheat Aegilops tauschii Coss. has extensive natural variation available for breeding of common wheat. Drought stress tolerance is closely related to abscisic acid (ABA) sensitivity. In this study, 17 synthetic hexaploid wheat lines, produced by crossing the tetraploid wheat cultivar Langdon with 17 accessions of Ae. tauschii, were used for comparative analysis of natural variation in drought tolerance and ABA sensitivity. Ae. tauschii showed wide natural variation, with weak association between the traits. Droughtsensitive accessions of Ae. tauschii exhibited significantly less ABA sensitivity. D-genome variations observed at the diploid genome level were not necessarily reflected in synthetic wheats. However, synthetic wheats derived from the parental Ae. tauschii accessions with high drought tolerance were significantly more tolerant to drought stress than those from drought-sensitive accessions. Moreover, synthetic wheats with high drought tolerance showed significantly higher ABA sensitivity than drought-sensitive synthetic lines. In the hexaploid genetic background, therefore, weak association of ABA sensitivity with drought tolerance was observed. To study differences in gene expression patterns between stress-tolerant and -sensitive lines, levels of two Cor/Lea and three transcription factor gene transcripts were compared. The more tolerant accession of Ae. tauschii tended to accumulate more abundant transcripts of the examined genes than the sensitive accession under stress conditions. The expression patterns in the synthetic wheats seemed to be
\end{abstract}


additive for parental lines exposed to drought and ABA treatments. However, the transcript levels of transcription factor genes in the synthetic wheats did not necessarily correspond to the postulated levels based on expression in parental lines. Allopolyploidization altered the expression levels of the stress-responsive genes in synthetic wheats.

Keywords: abscisic acid; allopolyploidization; drought stress; natural variation; synthetic wheat

\section{Introduction}

Abiotic stress signal pathways have been studied using artificially generated mutants in the model plant Arabidopsis thaliana. However, naturally occurring variants still provide an important alternative source of genetic variation [1]. Recent progress in the analysis of natural variation in Arabidopsis demonstrates the usefulness of wild populations for identification of genetic loci accounting for naturally occurring variation in abiotic stress tolerance [2-4]. Naturally occurring variants are useful in determining which specific allelic variants exist in nature, where they might either be neutral or have a selective advantage under specific conditions [1].

Abiotic stresses such as drought, temperature and salinity reduce yield of common wheat (Triticum aestivum L.). Under abiotic stress conditions, plant cells undergo drastic physiological, biochemical and metabolic changes leading to the development of stress tolerance at the cellular level. One of the mechanisms behind development of stress tolerance is induction of the Cor (cold-responsive)/Lea (late-embryogenesis-abundant) gene family; COR/LEA proteins promote the development of stress tolerance by protecting cellular components from stress [5]. A number of Cor/Lea genes are not only responsive to low temperature but also to drought and abscisic acid (ABA). Many low temperatureand drought-inducible genes contain both C-repeat (CRT)/dehydration-responsive element (DRE) and ABA-responsive element (ABRE) motifs in their promoters, and these cis elements are considered to function independently. Expression of these Cor/Lea genes is regulated by major transcription factors in the $\mathrm{CBF} / \mathrm{DREB}$ and $\mathrm{AREB} / \mathrm{ABF}$ families under abiotic stress conditions such as low temperature and osmotic stress [6].

ABA is one of the key plant hormones responding to environmental stress [7]. ABA is synthesized de novo mainly in response to drought and high salinity stress in vegetative tissues [8,9]. Several Cor/Lea genes, the expression of which correlate with increased freezing and drought tolerance, are also responsive to exogenous ABA [10], and ABRE sequences exist in the promoter regions of these Cor/Lea genes in Arabidopsis [11-13]. The Arabidopsis basic region/leucine zipper (bZIP)-type $\mathrm{AREB} / \mathrm{ABF}$ proteins bind as dimers to ABRE and activate ABA-dependent gene expression [14-16].

$\mathrm{CRT} / \mathrm{DRE}, \mathrm{ABRE}$ and other cold-responsive motifs have been identified in the 5' upstream regions of many wheat Cor/Lea genes such as Wdhn13 and Wrab17 [17-20]. Wheat CBF homologs such as TaCBF, TaDREB1 and WCBF2 encode transcription factors recognizing the CRT motif [21-24]. Of the $C B F$ homologs, TaDREB1 expression is clearly responsive to drought stress and ABA. In addition, wheat bZIP-type transcription factors, WABI5, TaOBF1 and WLIP19, bind to the Cor/Lea promoter regions and directly activate Cor/Lea gene expression [25,26]. Expression of WABI5, TaOBF1 and 
WLIP19 is increased after drought stress and ABA treatment in leaves of common wheat seedlings. Thus, Cor/Lea gene expression mediated by CBF/DREB and bZIP-type transcription factors might play a central role in development of abiotic stress tolerance in wheat.

Aegilops tauschii Coss. (syn. Ae. squarrosa Eig.), a wild relative of wheat, is one of the diploid progenitors of common wheat $[27,28]$. Common wheat is an allohexaploid species that originated from natural hybridization between tetraploid wheat (Triticum turgidum L.), including emmer and durum wheats, and Ae. tauschii [29,30]. Ae. tauschii is widely distributed in Eurasia and shows abundant genetic variation [31-36]. The birthplace of common wheat is considered to lie within the area comprising Transcaucasia and the southern coastal region of the Caspian Sea [37]. Therefore, the Ae. tauschii populations involved in the origin of common wheat are limited to a narrow distribution range relative to the entire species range, suggesting that this species holds vast genetic diversity that is not represented in common wheat [37]. Natural variation in the Ae. tauschii population provides potential for improving modern varieties of common wheat. In fact, hexaploid synthetic wheat derived from crosses between tetraploid wheat and Ae. tauschii has the potential to provide new genetic variation for abiotic stress tolerance [38].

In the present study, we surveyed naturally occurring genetic variation in drought tolerance and ABA sensitivity in Ae. tauschii. Next, we examined expression of the natural variation in synthetic hexaploid lines produced by crossing the tetraploid wheat cultivar Langdon with Ae. tauschii accessions. In addition, we compared expression of the Cor/Lea and Cor/Lea transcription factor genes to analyze the molecular nature of the natural variation in both synthetic wheats and their parental accessions. Based on these results, we discuss the relationship between drought tolerance and ABA sensitivity and the usefulness of Ae. tauschii variation in wheat breeding.

\section{Results}

\subsection{Natural Variation in Dehydration Tolerance and ABA Sensitivity in Aegilops Tauschii}

Thirty accessions of Ae. tauschii were examined to evaluate the level of drought tolerance and exogenous ABA sensitivity (Table 1). The drought tolerance level was calculated based on the survival rate of 10 -d-old seedlings after $24 \mathrm{~h}$ of drought treatment at $25^{\circ} \mathrm{C}$; it varied widely from $0 \pm 0.0 \%$ (KU2811) to $90.0 \pm 17.3 \%$ (KU-2093) in the 30 Ae. tauschii accessions (Figure 1A). All accessions from Iran $(n=7)$ showed high tolerance to drought stress, whereas Transcaucasus accessions exhibited comparatively low levels of drought tolerance. The average survival was $37.4 \pm 34.1 \%$. Thirteen of all the accessions examined showed less than 30\% survival, and only three accessions exhibited more than $70 \%$ survival under drought conditions (Figure 1B).

It was previously reported that cultivar difference of responsiveness to exogenous ABA was corresponding to the difference of abiotic stress tolerance in common wheat [19]. Here, we called the responsiveness to exogenous $\mathrm{ABA}$ as ABA sensitivity. ABA sensitivity was evaluated by inhibition of post-germination growth in the presence of $10 \mu \mathrm{M}$ ABA. ABA greatly reduced root length in most accessions of Ae. tauschii. The ABA sensitivity was measured by the relative growth inhibition rate (\% reduction in the presence $\mathrm{ABA}$ relative to growth in the absence of $\mathrm{ABA}$ ). 
Table 1. Origin and identity of Ae. tauschii accessions used in this study.

\begin{tabular}{|c|c|}
\hline Origin & Accession number \\
\hline Afghanistan & KU-2059ª, PI476874 ${ }^{\mathrm{b}}, \mathrm{KU}-2027$ \\
\hline Armenia & $\mathrm{KU}-2811^{\mathrm{a}}, \mathrm{KU}-2816$ \\
\hline Azerbaijan & IG47188, IG47193 \\
\hline China & $\mathrm{PI} 499262^{\mathrm{b}}, \mathrm{AT}_{4} 7^{\mathrm{c}}, \mathrm{AT}^{\mathrm{c}} 6^{\mathrm{c}}$ \\
\hline Dagestan & KU-20-1, IG120866 \\
\hline Georgia & $\mathrm{AE} 454^{\mathrm{c}}, \mathrm{KU}-2829 \mathrm{~A}^{\mathrm{c}}$ \\
\hline Kazakhstan & AE1090 \\
\hline Kyrgyzstan & IG131606 ${ }^{\mathrm{c}}$ \\
\hline India & $\mathrm{IG} 48042^{\mathrm{c}}$ \\
\hline Iran & KU-2076 ${ }^{\mathrm{b}}, \mathrm{KU}-2078^{\mathrm{a}}, \mathrm{KU}-2093^{\mathrm{b}}, \mathrm{KU}-2126^{\mathrm{b}}, \mathrm{KU}-2160^{\mathrm{c}}, \mathrm{KU}-2069, \mathrm{KU}-2088$ \\
\hline Pakistan & 皮 \\
\hline Syria & IG46623, IG47259 \\
\hline Tajikistan & IG $48554^{\mathrm{a}}$ \\
\hline Turkmenistan & IG48518, IG126387 \\
\hline
\end{tabular}

KU: Plant Germ-Plasm Institute, Faculty of Agriculture, Kyoto University, Japan.

PI: National Small Grains Research Facility, USDA-ARS, USA.

IG: International Centre for Agricultural Research in the Dry Areas (ICARDA), Syria.

AE: Institut für Pflanzengenetik und Kulturpflanzenforschung (IPK), Germany.

AT: Faculty of Agriculture, Okayama University, Japan.

a: Parental accessions for synthetic hexaploids produced in this study.

${ }^{\mathrm{b}}$ : Parental accessions for synthetic hexaploids reported in Takumi et al. [50].

c: Parental accessions for synthetic hexaploids from triploids in Matsuoka et al. [33].

It also varied widely from $2.0 \pm 7.3 \%$ (KU-2811) to $65.4 \pm 22.4 \%$ (KU-2160) in the 30 Ae. tauschii accessions (Figure. 1C, 1D). The average ABA sensitivity was $29.1 \pm 21.1 \%$.

The ABA sensitivity of each of the 30 Ae. tauschii accessions was compared with its drought tolerance. KU-20-1 showed exceptionally low drought tolerance in spite of high ABA sensitivity. In the 29 other accessions, the correlation between drought tolerance and ABA sensitivity was positive $\left(R^{2}=0.0993\right)$, but not significant (Figure 2A). These 29 accessions were classified into three groups: drought-sensitive $(<20 \%$ survival rate), moderately tolerant $(20-50 \%)$ and highly tolerant $(>50 \%)$ groups. Student's t-test showed that the ABA sensitivity of the drought-sensitive group was significantly $(P<0.05)$ lower than that of the moderately and highly tolerant groups (Figure $2 \mathrm{~B})$.

\subsection{Expression of Dehydartion Tolerance and ABA Sensitivity in Synthetic Wheats}

Seventeen lines of synthetic wheats were used to evaluate the level of drought tolerance and ABA sensitivity. These 17 lines were derived from interspecific crosses between a tetraploid wheat accession Triticum durum cv. Langdon and 17 Ae. tauschii accessions (Table 1). The somatic chromosome number was 42 in all $\mathrm{F}_{3}$ seeds examined from single $\mathrm{F}_{2}$ plants of each synthetic. All the synthetics were highly fertile and there was no segregation of plants with excessively reduced height (indicative of haploidy or aneuploidy) in the $F_{2}$ populations (data not shown), suggesting that the synthetics were hexaploid and stable. 
Figure 1. Natural variation in drought tolerance and ABA sensitivity in 30 Ae. tauschii accessions.

(A)

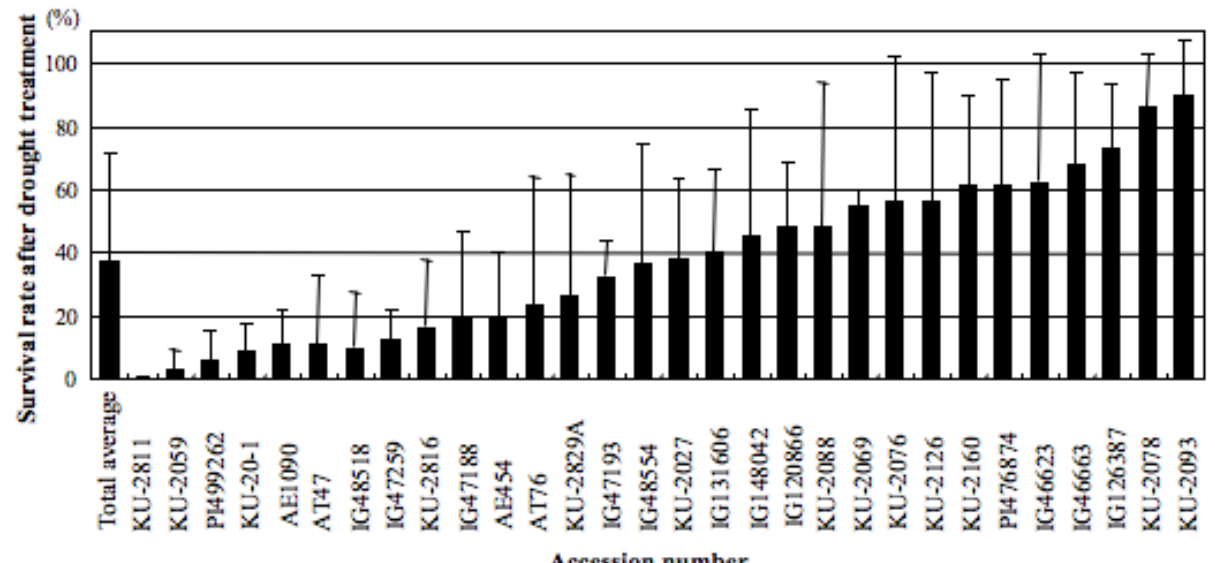

(B)

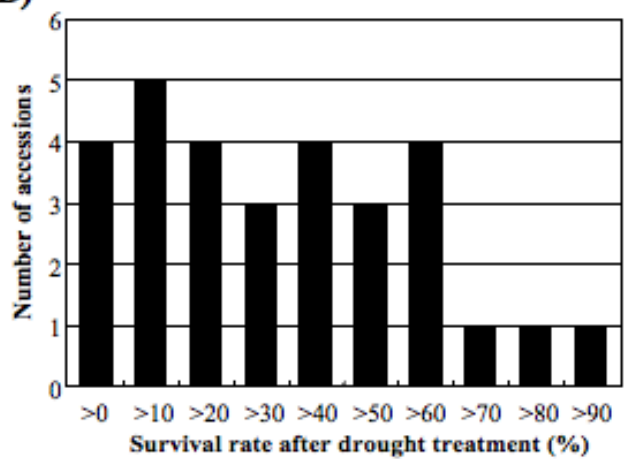

(D)

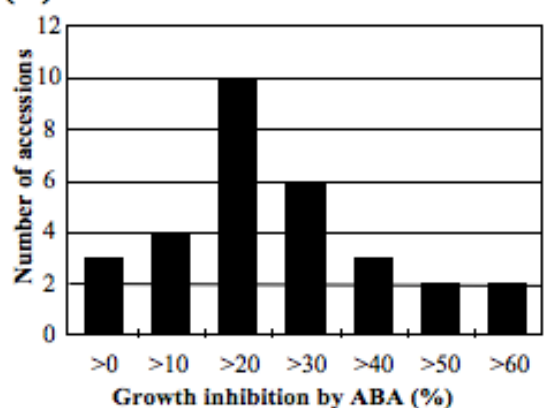

(C) $(\%)$

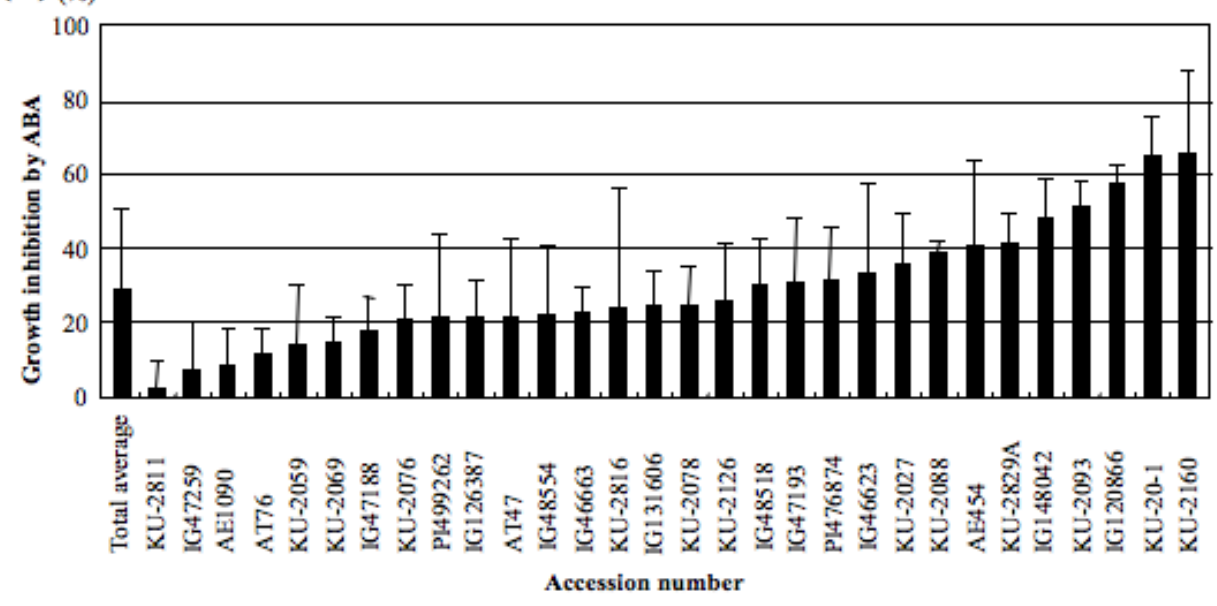

(A) Survival rates (\%) after $24 \mathrm{~h}$ drought stress. Means \pm SDs were calculated from data from three independent experiments. In each experiment, at least 10 plants were tested. (B) Frequency distribution of the drought tolerance levels (\%) in the 30 Ae. tauschii accessions. (C) Growth inhibition rate (\%) in the presence of $10 \mu \mathrm{M}$ ABA. Means \pm SD were calculated from data from three independent experiments. In each experiment, at least five plants were tested. (D) Frequency distribution of ABA sensitivity in the 30 Ae. tauschii accessions. 
Figure 2. Correlation between drought tolerance and ABA sensitivity in Ae. tauschii.

(A)

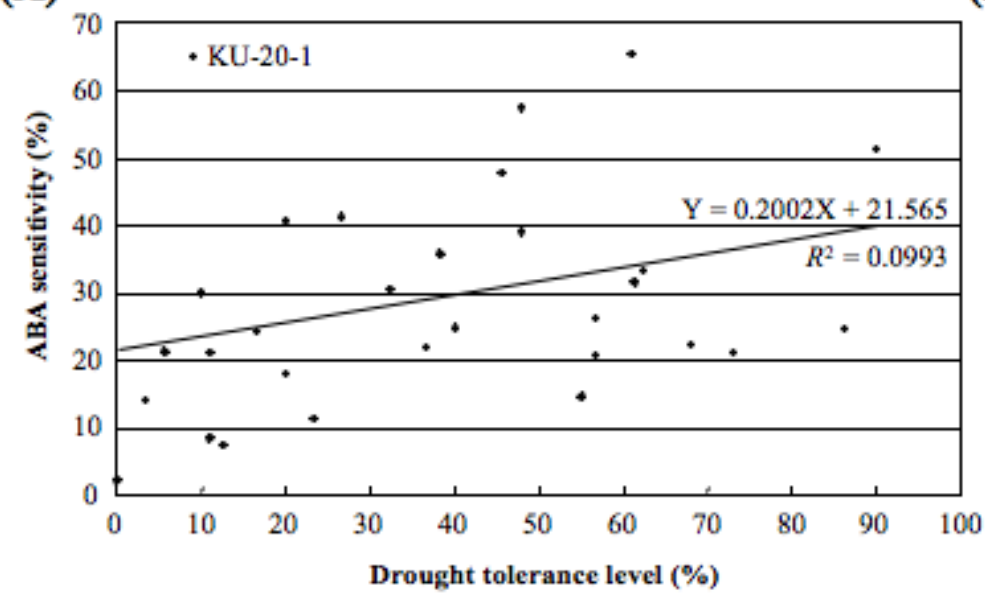

(B)

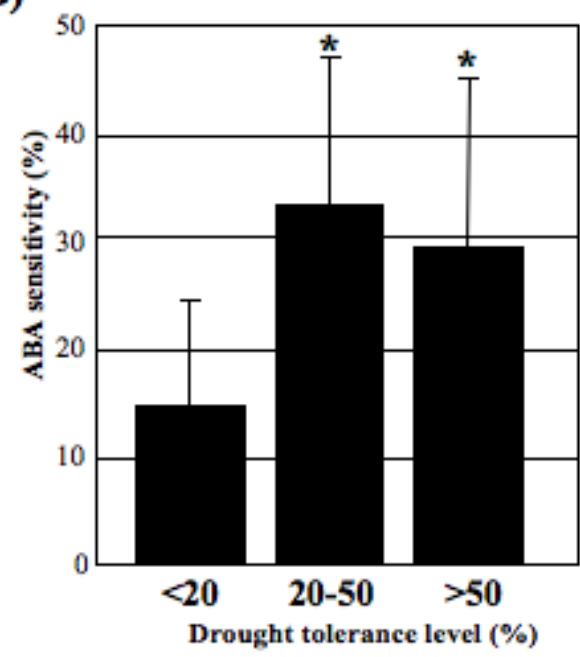

(A) Scatter plot showing drought tolerance and ABA sensitivity in 30 Ae. tauschii accessions. (B) Comparison of ABA sensitivity for three Ae. tauschii groups (excluding KU-20-1, which had a distinct level of drought tolerance). Student's $t$-test was used to test for statistical significance $\left({ }^{*} P<\right.$ 0.05 ) between the different categories of drought tolerance.

Seedlings of the synthetic wheat lines were treated by dehydration stress for four days, because the synthetic wheats were tolerant to the 24-h drought treatment. The synthetic wheats showed wide variation for both drought tolerance and sensitivity to exogenous $\mathrm{ABA}$, but the range of variation in the synthetic wheats was narrower than in the parental Ae. tauschii accessions (Figures 3A, 3D). The average drought tolerance level was $38.3 \pm 11.3 \%$ in synthetic wheats, and the tolerance levels varied from $16.7 \pm 5.8 \%$ (Langdon $x$ PI499262) to $56.7 \pm 25.2 \%$ (Langdon x KU-2160). There was a positive correlation of drought tolerance levels between synthetic wheats and the parental Ae. tauschii accessions $\left(R^{2}=0.238\right)$, but the correlation was not significant $(P=0.24)$ (Figure $\left.3 \mathrm{~B}\right)$. The 17 synthetic lines were divided into three groups based on the level of drought tolerance of their parental Ae. tauschii accessions. Group A consisted of four lines, of which the parental Ae. tauschii accessions PI499262, KU-2059, KU-2811 and AT47 showed drought sensitivity (less than 15\% survival rates). A total of six synthetic wheats were classified into group B and seven into group $\mathrm{C}$, for which the respective parental Ae. tauschii accessions exhibited moderate (20-50\%) and high (>50\%) drought tolerance levels. The group $\mathrm{C}$ lines showed significantly higher drought tolerance than group A (Figure 3C). In group $C$, the synthetic lines were more tolerant to drought stress than the parental Langdon (Figure 3A). 
Figure 3. Variation in drought tolerance and ABA sensitivity of 17 synthetic wheats and their parental lines.

(A)

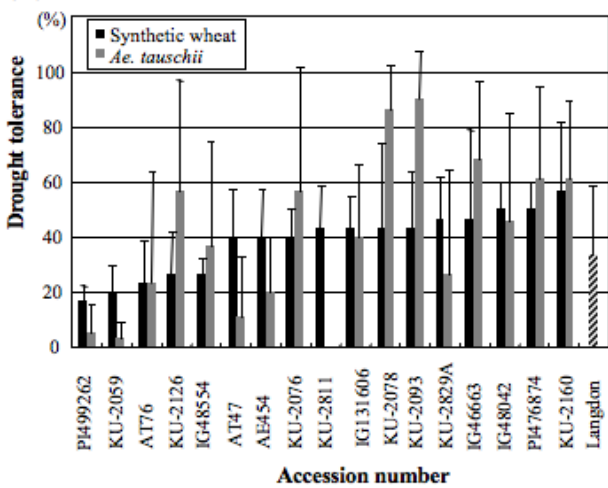

(B)

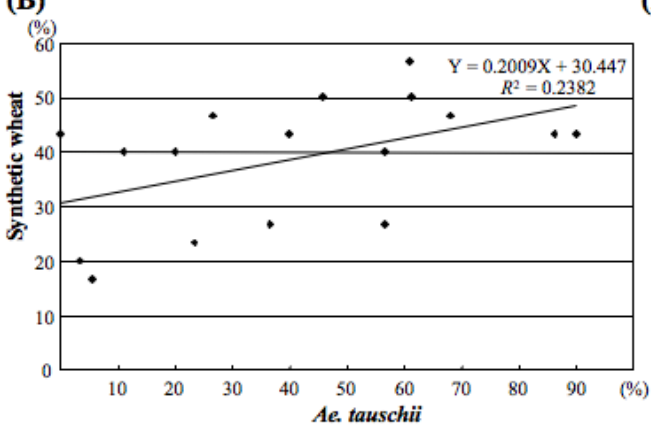

(C)

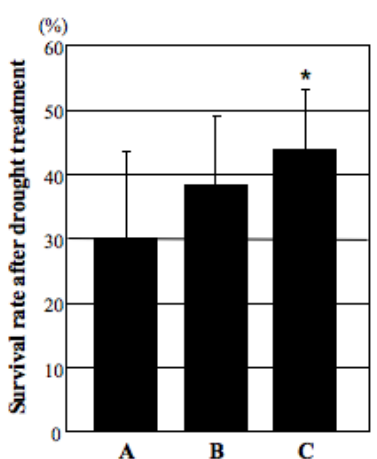

(D)
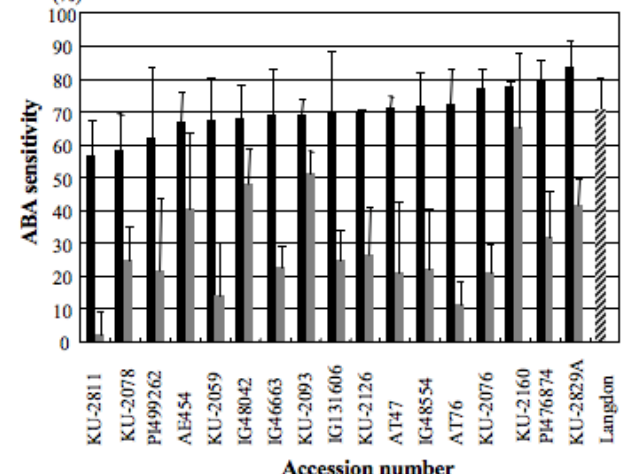

(E)

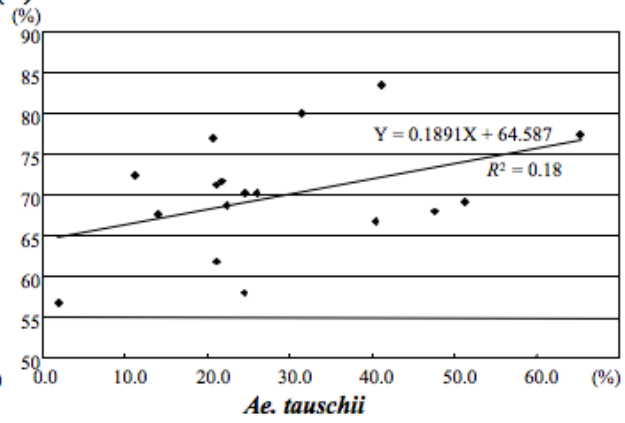

(F)

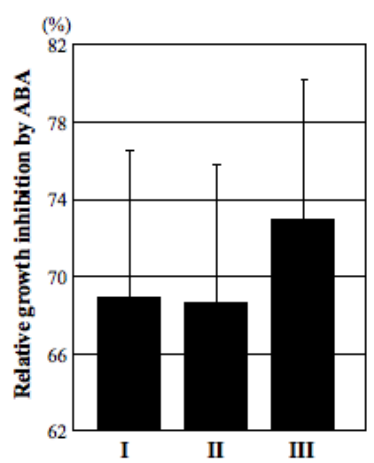

(A) Drought tolerance revealed by survival rate after a $4 \mathrm{~d}$ drought treatment. (B) Scatter plot of drought tolerance in the synthetics and parental Ae. tauschii accessions. (C) Comparison of drought tolerance for three categories of synthetics having parental Ae. tauschi lines with distinct levels of drought tolerance. Student's $t$-test was used to test for statistical significance $\left({ }^{*} P<0.05\right)$ compared with the drought-sensitive group with low drought tolerance. A, drought-sensitive accessions; B, accessions with moderate drought tolerance; $C$, highly drought tolerant accessions. (D) ABA sensitivity based on relative growth inhibition (\%) due to $20 \mu \mathrm{M}$ ABA treatment. Means \pm SD were calculated from data from three independent experiments. In each experiment, at least five plants were tested. (E) Scatter plot of ABA sensitivity in the synthetics and parental Ae. tauschii accessions. (F) Comparison of ABA sensitivity for three categories of synthetics having parental Ae. tauschi lines with distinct levels of ABA sensitivity. I, low ABA-sensitivity accessions; II, moderately ABA-sensitive accessions; III, highly ABA-sensitive accessions. 
Figure 4. Correlation between drought tolerance and ABA sensitivity in synthetic wheats and parental accessions.

(A)

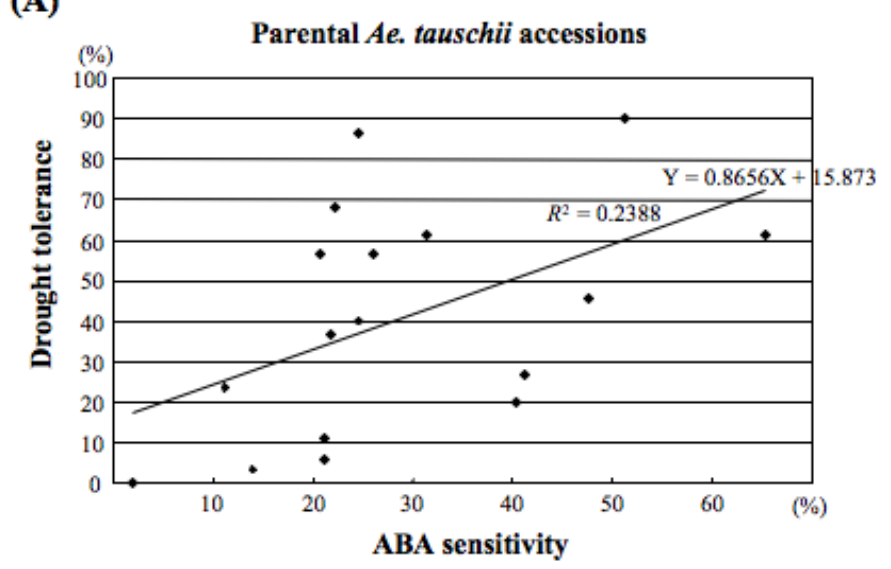

(B)

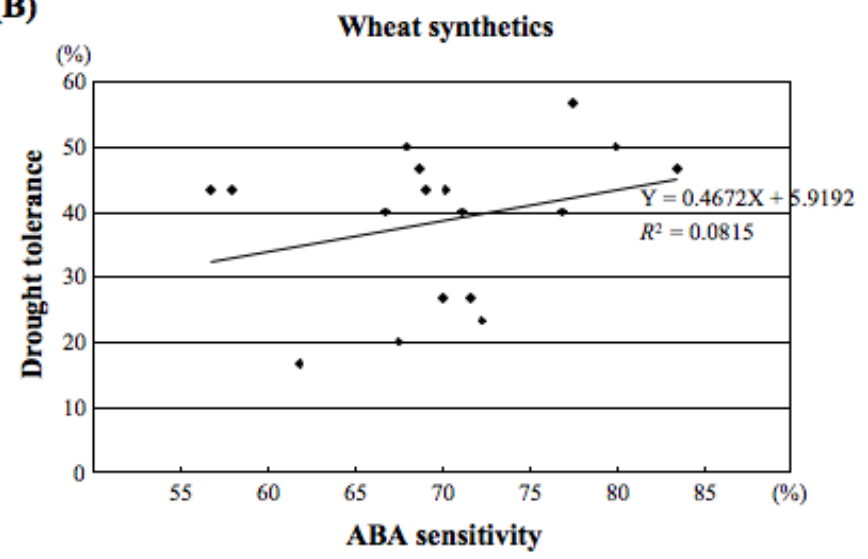

(C)

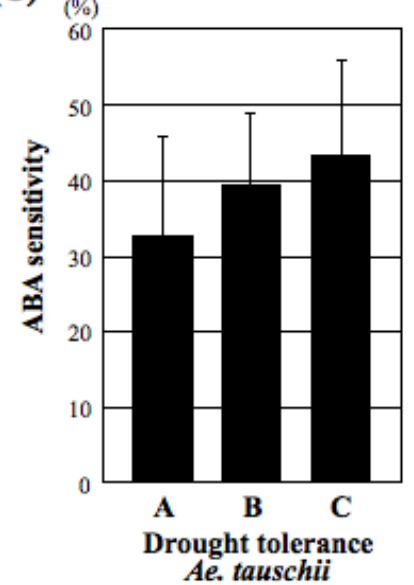

(D)

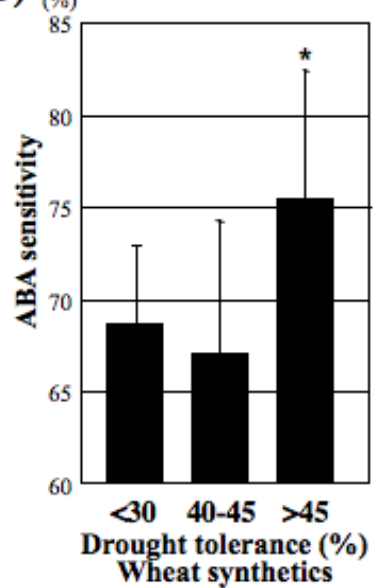

(A) Scatter plot of drought tolerance and ABA sensitivity in the parental Ae. tauschii accessions. (B) Scatter plot of drought tolerance and ABA sensitivity in the synthetic lines. (C) Comparison of ABA sensitivity for three groups of Ae. tauschii accessions with distinct levels of drought tolerance. A, drought-sensitive accessions; $\mathrm{B}$, accessions with moderate drought tolerance; $\mathrm{C}$, highly drought-tolerant group. (D) Comparison of ABA sensitivity for three groups of synthetic wheats with distinct levels of drought tolerance. Student's $t$-test was used to test for statistical significance $(* P<0.05)$ compared with the $>45 \%$ survival group with low drought tolerance. 
The average ABA sensitivity was $70.3 \pm 6.9 \%$ in synthetic wheats, and varied from $56.7 \pm 10.6 \%$ (Langdon $x$ KU-2811) to $83.5 \pm 8.2 \%$ (Langdon $x$ KU-2829A) under the $20 \mu \mathrm{M}$ ABA condition. The correlation $(R=0.424)$ of the ABA sensitivity between synthetic wheats and parental Ae. tauschii accessions was not significant $(P=0.09)$ (Figure 3E). The 17 synthetic wheats were classified into three groups based on the ABA sensitivities of their parental Ae. tauschii accessions. Five synthetic lines with less than $21 \%$ of their parental Ae. tauschii sensitivity belonged to group I and seven with 21 $40 \%$ sensitivity belonged to group II. Group III consisted of five accessions with more than $40 \%$ the ABA sensitivity of their parental Ae. tauschii accessions. No significant difference, however, was observed among the three groups. Although all parental Ae. tauschii accessions showed lower ABA sensitivity than Langdon, seven synthetic wheat lines were more sensitive to exogenous ABA than the parental Langdon (Figure 3D).

The relationship between the level of drought tolerance level and ABA sensitivity was studied in the hexaploid genetic background. Correlation $(R)$ values between drought tolerance and ABA sensitivity were $0.49(P=0.122)$ in the parental Ae. tauschii accessions and $0.29(P=0.26)$ in the synthetic wheats (Figures 4A, 4B). No significance was observed between the drought tolerance level and ABA sensitivity at either the diploid or hexaploid level. The range of variation in the level of drought tolerance and $\mathrm{ABA}$ sensitivity was narrower in the synthetic lines than in the parental Ae. tauschii accessions. The ABA sensitivity of the 17 synthetic wheat lines was also compared with the level of drought tolerance of both parental Ae. tauschii accessions and synthetic lines. No significant difference in ABA sensitivity was observed among the three groups based on the drought tolerance of the parental Ae. tauschii accessions (Figure 4C). The highly drought-tolerant group with more than $45 \%$ tolerance in the synthetic wheats was significantly more sensitive to exogenous ABA treatment than the other groups (Figure 4D).

\subsection{Expression Patterns of Cor/Lea Genes under Stress Conditions}

To compare gene expression patterns between lines with low and high ABA sensitivity, two Ae. tauschii accessions, KU-2811 (low ABA sensitivity) and KU-2829A (high ABA sensitivity), and synthetic lines derived from them were selected. Both KU-2811 and a synthetic wheat line derived from Langdon and KU-2811 showed the lowest ABA sensitivity, while KU-2811 was the most drought-sensitive. The ABA sensitivity of both KU-2829A and a synthetic wheat line derived from Langdon and KU-2829A was high, and the drought tolerance of the KU-2829A-derived synthetic line was also high.

Two Cor/Lea genes, Wrab17 and Wdhn13, are responsive to exogenous ABA and drought stress treatment [17-19]. Expression levels of the Actin gene used as an internal control were not changed by stress treatment in any of the accessions and lines examined. Wrab17 transcript accumulation was clearly induced by ABA and drought treatment in both Ae. tauschii accessions (Figure 5A). KU-2829A accumulated Wrab17 transcripts more abundantly than KU-2811 under both stress conditions. In Langdon and the two synthetic wheats, induction of Wrab17 expression was not clear, and the accumulation was highly abundant. No significant difference in expression pattern was observed for the $\mathrm{ABA}$ and drought treatments among Langdon and the two synthetic wheats. 
Figure 5. Expression patterns of two Cor/Lea genes (Wrab17 and Wdhn13) and three transcription factor genes (TaDREB1, WABI5 and TaOBF1) in the synthetic wheats, parental Ae. tauschii accessions and Langdon after ABA and drought-stress treatment.

\section{(A)}

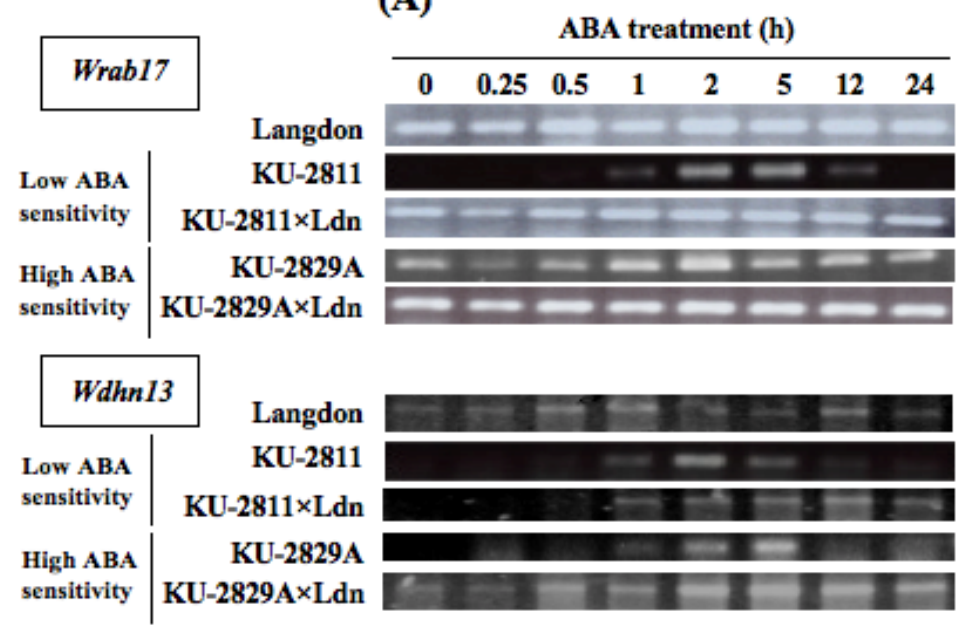

(B)
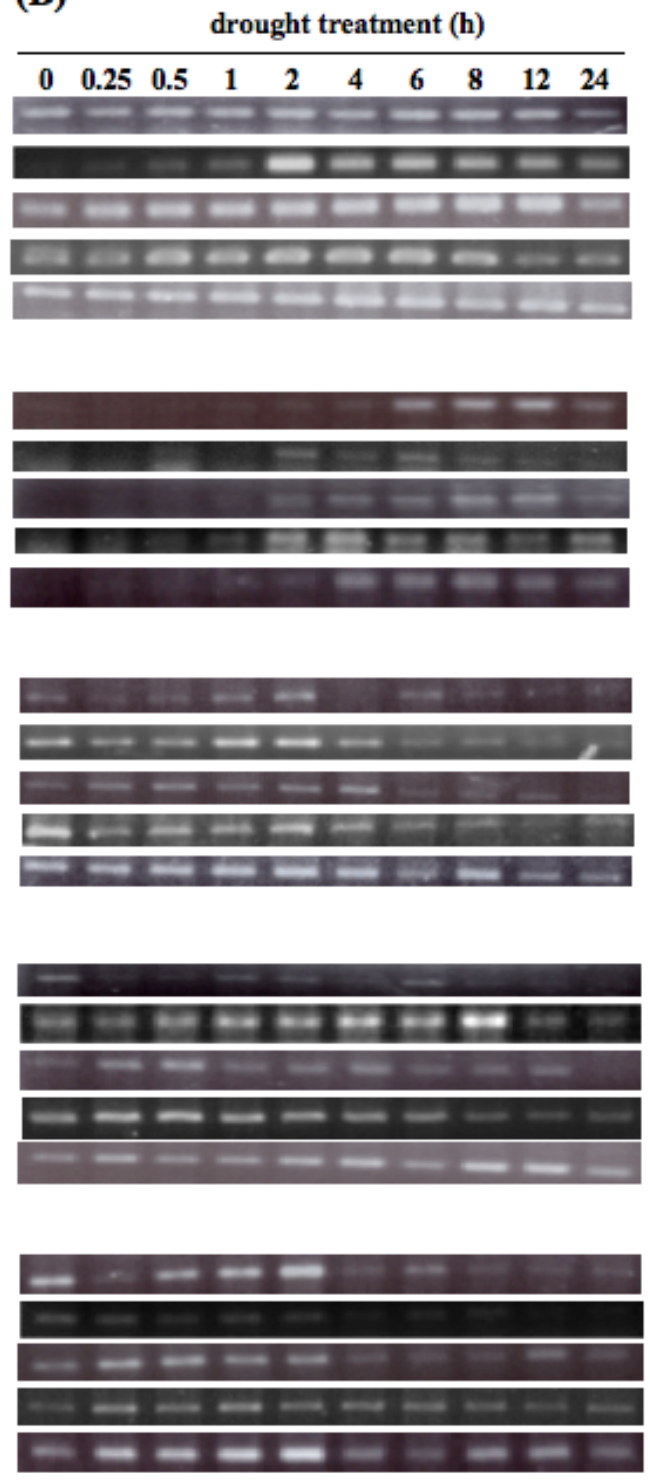

(A) ABA-responsive expression. Gene expression patterns were revealed by RT-PCR analysis using the same set of RNA preparations. Actin was used as internal control. Total RNA was extracted from leaves of seedlings after the indicated times in the $20 \mu \mathrm{M}$ ABA treatment. (B) Drought-responsive expression. Total RNA was extracted from leaves of seedlings after the indicated drought treatment. 
Figure 6. Comparison of transcript levels in synthetic wheats and their parental lines. Quantitative RT-PCR analysis was conducted using leaves from ABA- and drought stresstreated seedlings. The postulated levels in the synthetic wheats were calculated as 2:1 ratio mixtures of the transcript levels of the parental Langdon and Ae. tauschii accessions. Each transcript level was represented as the value relative to the Langdon level at $0.5 \mathrm{~h}$.

(A)

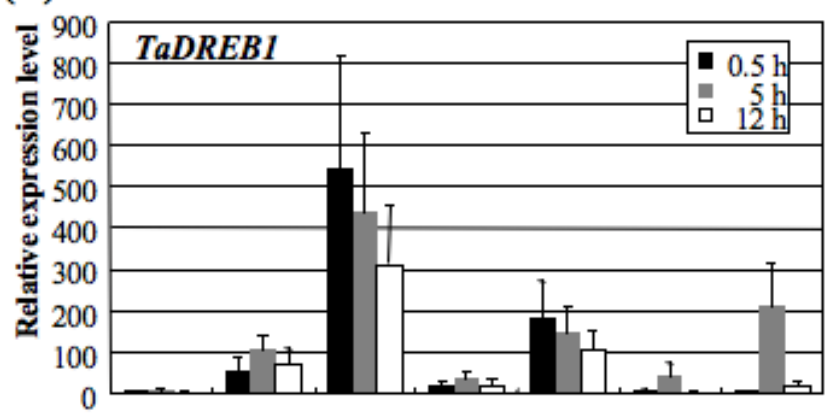

(C)

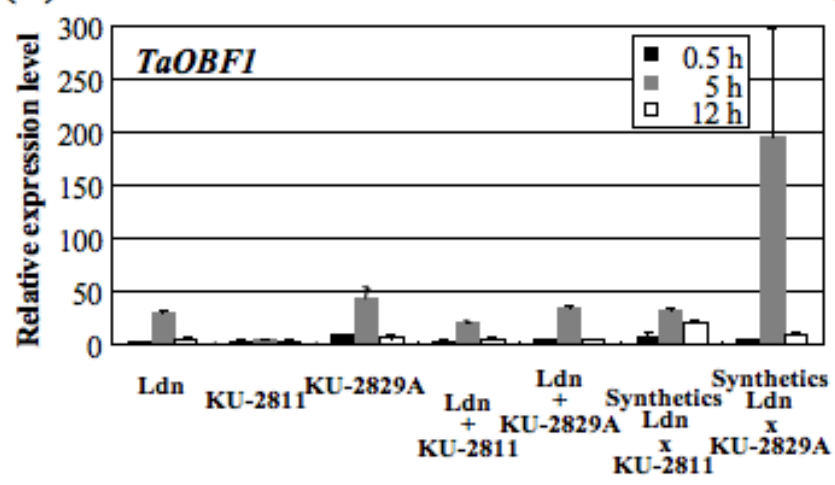

(B)

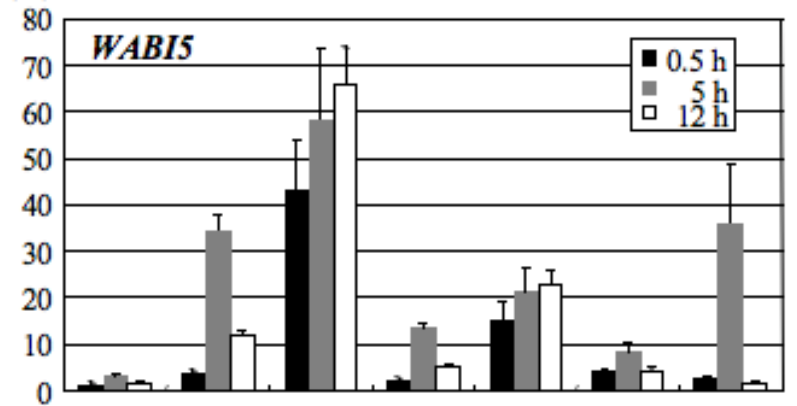

(D)

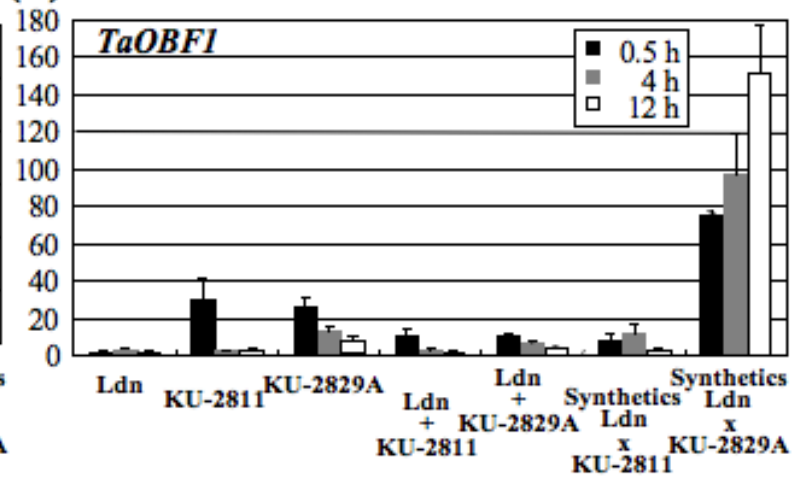

(A) TaDREB1 transcript levels after ABA treatment. (B) WABI5 transcript levels after ABA treatment. (C) TaOBF1 transcript levels after ABA treatment. (D) TaOBF1 transcript levels under drought conditions.

Wdhn13 expression was responsive to $\mathrm{ABA}$ and drought stress in all accessions and lines examined (Figure. 5B). The Wdhn13 transcript levels were higher in KU-2829A than in KU-2811 under the drought stress conditions. The ABA- and drought-responsive expression patterns in both synthetics seemed to correspond to the sum of those of Langdon and the parental Ae. tauschii accessions. ABAresponsive accumulation of Wdhn13 transcripts occurred earlier in the KU-2829A-derived synthetic line than in the KU-2811-derived line. Under drought stress, the Wdhn13 transcript level reached a high plateau earlier in the KU-2829A-derived synthetic line than in the KU-2811-derived line.

\subsection{Expression Patterns of Transcription Factor Genes under Stress Conditions}

In previous studies, we reported that Wrab17 and Wdhn13 expression is at least partly controlled by CBF/DREB-related transcription factors as well as by WABI5, WLIP19 and TaOBF1 transcription factors $[19,20,24,25]$. In the present study, expression patterns of three transcription factor genes, TaDREB1, WABI5 and TaOBF1, were compared for lines with low and very high ABA sensitivity. The RNA samples used for the analysis of expression of these transcription factor genes were the same ones used for profiling Wrab17 and Wdhn13 gene expression. 
Expression of TaDREB1, WABI5 and TaOBF1 was responsive to exogenous ABA treatment in Langdon and the parental Ae. tauschii accessions (Figure 5A). KU-2829A more abundantly accumulated TaDREB1 and WABI5 transcripts than KU-2811 after ABA treatment. TaDREB1 and WABI5 transcript levels were higher in the KU-2829A-derived synthetic line than in the KU-2811derived line. Expression of the three transcription factor genes was responsive to drought stress treatment, but the induction was more rapid than after ABA treatment (Figure 5B). The droughtresponsive expression patterns of TaDREB1, WABI5 and TaOBF1 in both synthetics seemed to correspond to the sum of those of Langdon and the parental Ae. tauschii accessions.

For quantitative comparison of transcript levels of TaDREB1, WABI5 and TaOBF1, real-time RTPCR analysis was conducted using RNA samples obtained after exogenous ABA (0.5, 5 and $12 \mathrm{~h})$ and drought $(0.5,4$ and $12 \mathrm{~h})$ treatments. Transcript levels relative to Langdon $(0.5 \mathrm{~h})$ were calculated for both the parental Ae. tauschii accessions and the synthetic wheat lines. The transcript levels in the synthetic wheats were also compared with the postulated transcript levels, which were calculated as a 2:1 mixture of Langdon and the parental Ae. tauschii accession. Transcript levels of TaDREB1, WABI5 and TaOBF1 in KU-2829A were much higher than those in KU-2811 after ABA treatment (Figure 6). The TaDREB1, WABI5 and TaOBF1 transcript levels in the KU-2829A-derived synthetic line were also higher than those in the KU-2811-derived line, but they were different than the postulated levels. Under drought stress conditions, TaOBF1 transcript in KU-2829A and the KU-2829A-derived synthetic line accumulated more abundantly than in KU-2811 and the KU-2811-derived synthetic line. Similarly to the expression levels after ABA treatment, the TaOBF1 transcript levels in the synthetic lines differed from the postulated levels.

\section{Discussion}

Naturally occurring genetic variation is one of the most important basic resources for plant biology [1]. Ae. tauschii has a wide natural species range in central Eurasia, ranging from northern Syria and Turkey to Western China. In previous studies, the natural variation of Ae. tauschii populations was analyzed for several traits, including flowering and morphological traits and hybrid lethality in crosses with tetraploid wheat [33-35]. Ae. tauschii is also known as the D-genome progenitor of hexaploid bread wheat. Therefore, hexaploid synthetic wheat lines can be artificially produced through allopolyploidization between tetraploid wheat and Ae. tauschii [39,40], implying that agronomically important genes from natural variation of the Ae. tauschii population are available for wheat breeding by making synthetic wheats. In this study, natural variation in drought tolerance level and ABA sensitivity was evaluated in the Ae. tauschii population. ABA is a plant hormone known to be tightly associated with environmental stress such as drought and salt [7]. Ae. tauschii showed wide natural variation for the level of drought tolerance and ABA sensitivity (Figure 1), and weak association between the two traits was observed (Figure 2). Drought-sensitive accessions of Ae. tauschii exhibited significantly less ABA sensitivity. This observation agreed with previous knowledge of the function of ABA in abiotic stress responses. Therefore, the natural variation analysis in this study suggested that ABA plays at least an important partial role in development of drought stress tolerance in wild diploid wheat. However, the correlation between the level of drought tolerance and ABA sensitivity was low in the Ae. tauschii accessions. Seven accessions of Ae. tauschii from Iran, mostly originating from the 
Caspian coastal region, showed high levels of drought tolerance. Drought-sensitive accessions of Ae. tauschii were distributed over a wide area including Syria, the Transcaucasus, Afghanistan and China. The correlation of drought stress response with geographical origin of the accessions was not necessarily found for ABA sensitivity. The low correlation value might be due to the presence of other factors contributing to drought stress tolerance or to methodological problems used to assess the level of drought tolerance. In this study, the survival rate after drought-stress treatment was used as a measure of the drought tolerance level. Other bioassay systems should also be examined to evaluate drought tolerance.

The 17 synthetic hexaploids used in this study were derived through endoreduplication forming triploid gametes in the interspecific ABD hybrids obtained by crossing Langdon with 17 of the Ae. tauschii accessions. Thus, the genetic variation among the lines fundamentally originated from the D genome because the A and B genomes of the synthetics were all derived from the same genotype. All 17 lines were somatically stable with $2 \mathrm{n}=42$ and did not show any abnormal phenotype, such as hybrid weakness or hybrid lethality, often observed in triploid hybrids between tetraploid wheat and Ae. tauschii $[33,41,42]$. Therefore, these 17 synthetic wheat lines provide a large potential not only for use in wheat breeding but also in the study of transmission and expression of genetic variation in the D genome in a hexaploid genomic background. In this study, large variation was observed in the level of drought tolerance and ABA sensitivity among these synthetics. For both traits, correlation between the synthetic wheats and parental Ae. tauschii accessions was positive, but not statistically significant. Therefore, the D-genome variations observed at the diploid genome level were not necessarily reflected in the synthetic wheats. However, the synthetic wheats derived from the parental Ae. tauschii accessions with high drought tolerance were significantly more tolerant to drought stress than those from the drought-sensitive accessions (Figure 3C). Moreover, the synthetic wheats with high drought tolerance showed significantly higher ABA sensitivity than the drought-sensitive synthetic lines (Figure 4D). In the hexaploid genetic background, therefore, some association of ABA sensitivity with drought tolerance could be observed. These results indicated that Ae. tauschii accessions with high levels of abiotic stress tolerance are good candidates for genetic resources to breed abiotic stresstolerant cultivars of bread wheat.

Two Cor/Lea genes, Wrab17 and Wdhn13, were responsive to exogenous ABA and drought-stress treatment in Ae. tauschii as well as common wheat [19]. In common wheat, these genes are also coldinducible, and the timing and level of induction are correlated with the level of freezing tolerance under low temperature in two cultivars with contrasting levels of freezing tolerance [43]. Similarly, KU-2829A, with high ABA sensitivity and drought tolerance, showed more rapid response in Cor/Lea gene expression than the ABA-insensitive and drought-sensitive KU-2811 (Figure 5). Although growth inhibition following ABA treatment was dramatic in KU-2811, for unknown reasons, Cor/Lea expression was ABA-responsive. The ABA responsiveness of KU-2811 seems to be quite low in the growth response but not completely disrupted in the gene response, suggesting that Wrab17 and Wdhn13 are involved in downstream genes functioning in the ABA-dependent signal pathway for development of drought tolerance. In the 5' upstream regions of Wrab17 and Wdhn13, several ciselements, such as CRT/DRE and ABRE, were recognized in our previous study [20]. TaDREB1 is a candidate for the transcription factor of Wrab17 and Wdhn13 [22], and WABI5 and TaOBF1 directly activate Wrab17 and Wdhn13 expression in common wheat $[25,26]$. The more tolerant accession KU- 
2829A tended to accumulate transcripts of the transcription factor genes more abundantly than the sensitive accession KU-2811 under stress conditions (Figure 6). These results indicated that the differences in abiotic stress responses between the two Ae. tauschii accessions might be due to further upstream genetic factor(s) in the ABA-dependent signal pathway. In Arabidopsis, the Versailles core collection contains significant natural variation in freezing tolerance, $\mathrm{CBF}$ gene sequences and $C B F$ and Cor/Lea gene expression patterns [44]. Although there tends to be more CBF and Cor gene expression in freezing-tolerant accessions, neither $C B F$ nor Cor gene expression is closely correlated with freezing tolerance, and the $C B F$ genes alone cannot explain all differences in the level of freezing tolerance [44], which implies difficulties in assessing the function of single transcription factors. Major genetic loci resulting in phenotypic differences in drought tolerance among the Ae. tauschii accessions should be identified via a mapping-based approach.

Allopolyploidization alters gene expression profiles in allopolyploid cells [45]. Gene expression patterns are stochastically and epigenetically changed during the generation of allopolyploid plants [46-48]. Synthetic wheat lines are powerful tools to study the effects of polyploidization on gene expression [49]. For example, a significant change in the WDREB2 gene expression pattern has been reported in hexaploid synthetic wheats [50]. Our RT-PCR analysis of Cor/Lea genes and the genes encoding their transcription factors indicated that their expression patterns in the hexaploid synthetics seemed to be additive of those in their parental lines under both exogenous ABA treatment and drought conditions (Figure 5). However, real-time RT-PCR analysis demonstrated that accumulation of transcripts of transcription factor genes in the hexaploid synthetics did not necessarily correspond to the postulated levels based on expression in parental lines (Figure 6). These observations indicated that hexaploidization through production of the synthetic wheats altered the expression levels of ABA- and drought-responsive genes under stress conditions. It was quite difficult to evaluate the effect of the altered expression levels on stress tolerance. In fact, the drought tolerance levels of the synthetic wheats were generally higher than those of their parental Ae. tauschii accessions (Figure 3D). Alteration of gene expression profiles through allopolyploidization should be further studied in synthetic wheats using transcriptome-based approaches.

\section{Experimental Section}

\subsection{Plant Materials}

A total of 30 Ae. tauschii accessions representing the entire natural habitat range of the species was used in the study (Table 1). Passport data for these accessions, including the geographical coordinates of the original collection sites, have been given in Matsuoka et al. [33,34]. For each accession, we used seeds propagated from a single plant by self-pollination. Twelve hexaploid synthetic wheats previously reported $[33,51]$ were used, and five synthetic hexaploids were additionally produced in this study (Table 1). For production of the 17 synthetics, tetraploid wheat accession Triticum durum cv. Langdon was used as the female parent and crossed with each of the 17 Ae. tauschii accessions. The $F_{1}$ progeny were grown and selfed to produce synthetics (herein designated the $F_{2}$ generation). All 17 synthetics were independently generated through unreduced gamete formation in each of the triploid $\mathrm{F}_{1}$ hybrids [40]. The synthetics thus contained the A and B genomes from Langdon and the diverse $\mathrm{D}$ genomes originating from the Ae. tauschii male parents. All grew normally and showed 
none of the hybrid lethality or weakness, such as necrosis and chlorosis, often observed in triploid hybrids between tetraploid wheats and Ae. tauschii $[33,41,42]$. Somatic chromosome numbers were determined from root-tip mitotic preparations of three $F_{3}$ seeds from one $F_{2}$ plant of each synthetic, using the standard acetocarmine squash method.

\subsection{Bioassay for Abiotic Stress Tolerance}

Seedlings were grown under standard conditions $\left(23^{\circ} \mathrm{C}\right)$ according to Kobayashi et al. [43]. For analysis of drought tolerance, 10-day-old seedlings $(\mathrm{n}=20)$ of Ae. tauschii were removed from the soil and kept on dry filter paper for $24 \mathrm{~h}$ at $25^{\circ} \mathrm{C}$. Seven-day-old seedlings $(\mathrm{n}=20)$ of synthetic wheats $\left(\mathrm{F}_{3}\right.$ generation) were dehydrated on dry paper for $4 \mathrm{~d}$ at $23^{\circ} \mathrm{C}$. Stress-treated seedlings were transferred back to the standard conditions, and on the 7 th day after transfer, the number of surviving seedlings was recorded. To bioassay ABA sensitivity based on post-germination growth, seeds were imbibed under tap water for $5 \mathrm{~h}$ and kept overnight at $4^{\circ} \mathrm{C}$. Imbibed seeds were placed in a glass petri dish containing filter paper wetted with distilled water, and incubated for $24 \mathrm{~h}$ at $20^{\circ} \mathrm{C}$ in darkness. Ten synchronously germinated seeds were further treated with distilled water or 10 or $20 \mu \mathrm{M}$ ABA solution under the same conditions as the germination assay. After $3 \mathrm{~d}$, the length of primary roots was recorded. The whole experiment was repeated at least three times. The data were statistically analyzed using JMP software ver. 5.1.2 (SAS Institute). Correlations among the morphological traits were estimated based on Pearson correlation coefficient values.

\subsection{Expression Analysis}

Total RNA was extracted from the leaves of 7-d-old seedlings for various times under stress conditions. Seven-day-old seedlings were also treated with a solution containing ABA $(20 \mu \mathrm{M})$ by a foliar spray or were dehydrated on dry filter paper in a desiccator. The transcript accumulation of Cor/Lea genes such as Wdhn13 and Wrab17 and their three transcription factor genes, TaDREB1, WABI5 and TaOBF1, was detected by RT-PCR amplification as previously reported [19,20]. RT-PCR for TaDREB1 was conducted with the following gene-specific primer pair: 5'-AGTCTCCTCCTTCTC TTATCTC-3' and 5'-TTCTTGTACCCGTTGACTTATG-3'. The actin gene (Actin) was used as an internal control with the following gene-specific primer pair: 5'-GGCTGGTTTTGCTGGTGACGA AT-3' and 5'-AATGAAGGAAGGCTGGAAGAGGA-3'. The PCR products were separated by electrophoresis through a $1.5 \%$ agarose gel and stained with ethidium bromide for detection.

Quantitative RT-PCR was performed using a Thermal Cycler Dice ${ }^{\circledR}$ Real Time System (TakaraBio, Ohtsu, Japan) and gene-specific primer sets. For TaDREB1 and WABI5, the following two primer pairs were designed: 5'-TCTCTCTCGTCCCTCTTCTC-3' and 5'-TTTTCCTCCTTCCACTTCTT-3', and 5'-GGGATtGTGAGGGGGAGGAG-3' and 5'-GGCGGACTCCCTGTTCTTGA-3', respectively. For the other genes, the same primer pairs used for RT-PCR amplification were used in the quantitative RT-PCR. As an endogenous control, Actin was used. The rate of amplification was monitored by SYBR ${ }^{\circledR}$ Premix Ex Taq ${ }^{\mathrm{TM}}$ II (Takara-Bio) according to the manufacturer's protocol. Results were presented as $2^{-\Delta \mathrm{Ct}}$, where $\mathrm{Ct}$ is the number of PCR cycles required to reach the log phase of amplification for the examined genes minus the number of cycles to reach the same stage for Act, 
and then were represented as values relative to the transcript levels in samples of Langdon obtained at $0.5 \mathrm{~h}$.

\section{Conclusions}

A wild wheat progenitor, Ae. tauschii, had large genetic variation in drought tolerance and exogenous ABA sensitivity. The level of drought tolerance was at least partly related to ABA sensitivity. Similarly, synthetic wheats derived from hybrids between Langdon and Ae. tauschii accessions showed wide variations in drought tolerance and ABA sensitivity, although no significant correlation between the synthetic wheats and their parental Ae. tauschii accessions was observed. However, synthetic wheats derived from the parental Ae. tauschii accessions with high drought tolerance were significantly more tolerant to drought stress than those from the drought-sensitive accessions. Therefore, Ae. tauschii accessions with high levels of abiotic stress tolerance are expected to be useful resources to breed abiotic stress-tolerant cultivars of bread wheat. Allohexaploidization altered the expression profile of abiotic stress-responsive genes. Previous studies on the transcriptome in allopolyploids used a limited number of artificially produced lines. Therefore, further studies, including detailed systematic analyses, will be needed to explain the molecular basis of modifications of D-genome variation patterns following allopolyploidization.

\section{Acknowledgements}

We thank Drs. J. Valkoun (ICARDA), J. Konopka (ICARDA), H. Bockelman (USDA), A. Graqner (IPK), T. Kawahara (KU) and K. Kato (Okayama Univ.) for the Ae. tauschii accession seeds. We also thank Drs. Y. Matsuoka and F. Kobayashi for their kind help. This work was supported by a grant from the Ministry of Agriculture, Forestry and Fisheries of Japan (Genomics for Agricultural Innovation, TRC-1003).

\section{References and Notes}

1. Koornneef, M.; Alonso-Blanco, C.; Vreugdenhil, D. Naturally occurring genetic variation in Arabidopsis thaliana. Annu. Rev. Plant Biol. 2004, 55, 141-172.

2. Alonso-Blanco, C.; Gomez-Mena, C.; Llorente, F.; Koornneef, M.; Salinas, J.; Martinez-Zapater, J.M. Genetic and molecular analyses of natural variation indicate CBF2 as a candidate gene for underlying a freezing tolerance quantitative trait locus in Arabidopsis. Plant Physiol. 2005, 139, 1304-1312.

3. Hannah, M.A.; Wise, D.; Freund, S.; Fiehn, O.; Heyer, A.G.; Hincha, D.K. Natural genetic variation of freezing tolerance in Arabidopsis. Plant Physiol. 2006, 142, 98-112.

4. Bouchabke, O.; Chang, F.; Simon, M.; Voisin, R.; Pelletier, G.; Durand-Tardif, M. Natural variation in Arabidopsis thaliana as a tool for highlighting differential drought responses. PLoS ONE 2008, 3, e1705, doi:10.1371/journal.pone.0001705.

5. Thomashow, M.F. Plant cold acclimation: Freezing tolerance genes and regulatory mechanisms. Annu. Rev. Plant Physiol. Plant Mol. Biol. 1999, 50, 571-599. 
6. Yamaguchi-Shinozaki, K.; Shinozaki, K. Transcriptional regulatory networks in cellular responses and tolerance to dehydration and cold stresses. Annu. Rev. Plant Biol. 2006, 57, 781-803.

7. Leung, J.; Giraudat, J. Abscisic acid signal transduction. Annu. Rev. Plant Physiol. Plant Mol Biol. 1998, 49, 199-222.

8. Shinozaki, K.; Yamaguchi-Shinozaki, K.; Seki, M. Regulatory network of gene expression in the drought and cold responses. Curr. Opin. Plant Biol. 2003, 6, 410-417.

9. Xiong, L.; Zhu, J.K. Regulation of abscisic acid biosynthesis. Plant Physiol. 2003, 133, 29-36.

10. Palva, E.T. Gene expression under low temperature stress. In Stress Induced Gene Expression in Plants, Basra, A.S., Ed.; Harwood Academic Publishers: New York, NY, USA, 1994; pp. 103130.

11. Lång, V.; Palva, E.T. The expression of a rab-related gene, rab18, is induced by abscisic acid during the cold acclimation process of Arabidopsis thaliana (L.) Heynh. Plant Mol. Biol. 1992, 20, 951-962.

12. Baker, S.S.; Wilhelm, K.S.; Thomashow, M.F. The 5'-region of Arabidopsis thaliana cor15a has cis-acting elements that confer cold-, drought- and ABA-regulated gene expression. Plant Mol. Biol. 1994, 24, 701-713.

13. Yamaguchi-Shinozaki, K.; Shinozaki, K. A novel cis-acting element in an Arabidopsis gene is involved in responsiveness to drought, low-temperature, or high salt stress. Plant Cell 1994, 6, 251-264.

14. Choi, H.; Hong, J.; Ha, J.; Kang, J.; Kim, S.Y. ABFs, a family of ABA-responsive element binding factors. J. Biol. Chem. 2000, 275, 1723-1730.

15. Uno, Y.; Furihata, T.; Abe, H.; Yoshida, R.; Shinozaki, K.; Yamaguchi-Shinozaki, K. Arabidopsis basic leucine zipper transcription factors involved in an abscisic acid-dependent signal transduction pathway under drought and high-salinity conditions. Proc. Natl. Acad. Sci. USA 2000, 97, 11632-11637.

16. Kim, S.Y. The role of ABF family bZIP class transcription factors in stress response. Physiol. Plant 2006, 126, 519-527.

17. Tsuda, K.; Tsvetanov, S.; Takumi, S.; Mori, N.; Atanassov, A.; Nakamura, C. New members of a cold-responsive Lea/Rab-related Cor gene family from common wheat (Triticum aestivum L.). Genes Genet. Syst. 2000, 75, 179-188.

18. Ohno, R.; Takumi, S.; Nakamura, C. Kinetics of transcript and protein accumulation of a lowmolecular-weight wheat LEA D-11 dehydrin in response to low temperature. J. Plant Physiol. 2003, 160, 193-200.

19. Kobayashi, F.; Takumi, S.; Egawa, C.; Ishibashi, M.; Nakamura, C. Expression patterns of low temperature responsive genes in a dominant ABA-less-sensitive mutant line of common wheat. Physiol. Plant. 2006, 127, 612-623.

20. Kobayashi, F.; Ishibashi, M.; Takumi, S. Transcriptional activation of Cor/Lea genes and increase in abiotic stress tolerance through expression of a wheat DREB2 homolog in transgenic tobacco. Transgenic Res. 2008, 7, 755-767.

21. Jaglo, K.R.; Kleff, S.; Amundsen, K.L.; Zhang, X.; Haake, V.; Zhang, J.Z.; Deits, T.; Thomashow, M.F. Components of the Arabidopsis C-repeat/dehydration responsive element 
binding factor cold-responsive pathway are conserved in Brassica napus and other plant species. Plant Physiol. 2001, 127, 910-917.

22. Shen, Y.G.; Zhang, W.K.; He, S.J.; Zhang, J.S.; Liu, Q.; Chen, S.Y. An EREBP/AP2-type protein in Triticum aestivum was a DRE-binding transcription factor induced by cold, dehydration and ABA stress. Theor. Appl. Genet. 2003, 106, 923-300.

23. Kume, S.; Kobayashi, F.; Ishibashi, M.; Ohno, R.; Nakamura, C.; Takumi, S. Differential and coordinated expression of Cbf and Cor/Lea genes during long-term cold acclimation in two wheat cultivars showing distinct levels of freezing tolerance. Genes Genet. Syst. 2005, 80, 185-197.

24. Takumi, S.; Shimamura, C.; Kobayashi, F. Increased freezing tolerance through up-regulation of downstream genes via the wheat CBF gene in transgenic tobacco. Plant Physiol. Biochem. 2008, 46, 205-211.

25. Kobayashi, F.; Maeta, E.; Terashima, A.; Kawaura, K.; Ogihara, Y.; Takumi, S. Development of abiotic stress tolerance via bZIP-type transcription factor LIP19 in common wheat. J. Exp. Bot. 2008, 59, 891-905.

26. Kobayashi, F.; Maeta, E.; Terashima, A.; Takumi, S. Positive role of a wheat HvABI5 ortholog in abiotic stress response of seedlings. Physiol. Plant. 2008, 134, 74-86.

27. Eig, A. Monographisch-kritische Übersicht der Gatteung Aegilops. Repertorium Specierum Novarum Rgni Vegetabilis. Beihefte 1929, 55, 1-228.

28. Van Slageren, M.W. Wild Wheats: A Monograph of Aegilops L. and Amblyopyrum (Jaub. \& Spach) Eig (Poaceae), Wageningen Agricultural University Press: Wageningen, The Netherlands, 1994; pp. 326-344.

29. Kihara, H. Discovery of the DD-analyser, one of the ancestors of Triticum vulgare (in Japanese). Agric. Hortic. 1944, 19, 889-890.

30. McFadden, E.S.; Sears, E.R. The artificial synthesis of Triticum spelta. Rec. Genet. Soc. Am. 1994, 13, 26-27.

31. Dudnikov, A.J.; Goncharov, N.P. Allozyme variation in Aegilops squarrosa. Hereditas 1993, 119, 117-122.

32. Dvorak, J.; Luo, M.C.; Yang, Z.L.; Zhang, H.B. The structure of the Aegilops tauschii genepool and the evolution of hexaploid wheat. Theor. Appl. Genet. 1998, 97, 657-670.

33. Matsuoka, Y.; Takumi, S.; Kawahara, T. Natural variation for fertile triploid $F_{1}$ formation in allohexaploid wheat speciation. Theor. Appl. Genet. 2007, 115, 509-518.

34. Matsuoka, Y.; Takumi, S.; Kawahara, T. Flowering time diversification and dispersal in central Eurasian wild wheat Aegilops tauschii Coss.: genealogical and ecological framework. PLoS ONE 2008, 3, e3138, doi:10.1371/journal.pone.0003138.

35. Matsuoka, Y.; Nishioka, E.; Kawahara, T.; Takumi, S. Genealogical analysis of subspecies divergence and spikelet-shape diversification in central Eurasian wild wheat Aegilops tauschii Coss. Plant Syst. Evol. 2009, 279, 233-244.

36. Dudnikov, A.J.; Kawahara, T. Aegilops tauschii: genetic variation in Iran. Genet. Resour. Crop Evol. 2006, 53, 579-586.

37. Feldman, M. Origin of cultivated wheat. In The World Wheat Book: A History of Wheat Breeding, Bonjean, A.P., Angus, W.J., Eds.; Lavoisier Publishing: Paris, France, 2001; pp. 3-53. 
38. Trethowan, R.M.; Mujeeb-Kazi, A. Novel germplasm resources for improving environmental stress tolerance of hexaploid wheat. Crop Sci. 2008, 48, 1255-1265.

39. Kihara, H.; Lilienfeld, F. A new-synthesized 6x-wheat. Hereditas 1949, Suppl, 307-319.

40. Matsuoka, Y.; Nasuda, S. Durum wheat as a candidate for the unknown female progenitor of bread wheat: an empirical study with a highly fertile $\mathrm{F}_{1}$ hybrid with Aegilops tauschii Coss. Theor. Appl. Genet. 2004, 109, 1710-1717.

41. Nishikawa, K. Hybrid lethality in crosses between emmer wheats and Aegilops squarrosa. I. Vitality of $F_{1}$ hybrids between emmer wheats and Ae. squarrosa var. typica. Seiken Ziho 1960, $11,21-28$.

42. Nishikawa, K. Hybrid lethality in crosses between emmer wheats and Aegilops squarrosa. II. Synthetized 6x wheats employed as test varieties. Jpn. J. Genet. 1962, 37, 227-236.

43. Kobayashi, F.; Takumi, S.; Nakata, M.; Ohno, R.; Nakamura, T.; Nakamura, C. Comparative study of the expression profiles of the Cor/Lea gene family in two wheat cultivars with contrasting levels of freezing tolerance. Physiol. Plant 2004, 120, 585-594.

44. McKhann, H.I.; Gery, C.; Bérard, A.; Léveque, S.; Zuther, E.; Hincha, D.K.; Mita, S.D.; Brunel, D.; Téoulé, E. Natural variation in $\mathrm{CBF}$ gene sequences, gene expression and freezing tolerance in the Versailles core collection of Arabidopsis thaliana. BMC Plant Biol. 2008, 8, 105, doi:10.1186/1471-2229-8-105.

45. Comai, L. Genetic and epigenetic interactions in allopolyploid plant. Plant Mol. Biol. 2000, 43, 387-399.

46. Kashkush, K.; Feldman, M.; Levy, A.A. Gene loss, silencing and activation in a newly synthesized wheat allotetraploid. Genetics 2002, 160, 1651-1659.

47. Madlung, A.; Masuelli, R.W.; Watson, B.; Reynolds, S.H.; Davison, J.; Comai, L. Remodelling of DNA methylation and phenotypic and transcriptional changes in synthetic Arabidopsis allotetraploids. Plant Physiol. 2002, 129, 733-746.

48. Wang, J.; Tian, L.; Madlung, A.; Lee, H.S.; Chen, M.; Lee, J.J.; Watson, B.; Kagochi, T.; Comai, L.; Chen, Z.J. Stochastic and epigenetic changes of gene expression in Arabidopsis polyploids. Genetics 2004, 167, 1961-1973.

49. Pumphrey, M.; Bai, J.; Laudencia-Chingcuanco, D.; Anderson, O.; Gill, B. Nonadditive expression of homoeologous genes is established upon polyploidization in hexaploid wheat. Genetics 2009, 181, 1147-1157.

50. Terashima, A.; Takumi, S. Allopolyploidization reduces alternative splicing efficiency for transcripts of the wheat DREB2 homolog, WDREB2. Genome 2009, 52, 100-105.

51. Takumi, S.; Naka, Y.; Morihiro, H.; Matsuoka, Y. Expression of morphological and flowering time variation through allopolyploidization: an empirical study with 27 synthetic wheats and their parental Aegilops tauschii accessions. Plant Breed. 2009, 12, doi:10.1111/j.14390523.2009.01630.x.

(C) 2009 by the authors; licensee Molecular Diversity Preservation International, Basel, Switzerland. This article is an open-access article distributed under the terms and conditions of the Creative Commons Attribution license (http://creativecommons.org/licenses/by/3.0/). 